\title{
Wide Range Optical Studies on Transparent SWNT Films
}

\author{
F. Borondics*, K. Kamarás*, Z. Chen ${ }^{\dagger}$, A.G. Rinzler ${ }^{\dagger}$, M. Nikolou ${ }^{\dagger}$ and \\ D.B. Tanner ${ }^{\dagger}$ \\ * Research Institute for Solid State Physics and Optics, Hungarian Academy of Sciences, \\ P. O. Box 49, Budapest, H-1525, Hungary \\ ${ }^{\dagger}$ Department of Physics, University of Florida, Gainesville, FL 32611, U.S.A.
}

\begin{abstract}
We present transmission spectra from the far infrared through the ultraviolet region on freestanding SWNT films at temperatures between 40 and $300 \mathrm{~K}$. Several interesting features are observed in the low-frequency part of the spectrum: the Drude-like frequency dependence of the metallic tubes as well as a (sample-dependent) peak in the conductivity around $0.01 \mathrm{eV}$. We also studied the accidental nitrate doping of the SWNT samples during purification by nitric acid. Asprepared purified samples exhibit increased metallic absorption and decreased interband transitions; these features disappear on heating in vacuum.
\end{abstract}

\section{INTRODUCTION}

Frequency-dependent optical measurements on carbon nanotubes can render a wealth of information about their electronic structure. Early optical evidence for metallic (or semimetallic) carriers in single-wall carbon nanotubes came from reflectance spectra obtained in a wide temperature and frequency range [1]. Similar transmission studies on airbrushed nanotubes [2] and freestanding films [3] have not included temperature dependence. Here we present temperature-dependent spectra and the effect of accidental nitrate doping during purification, which influences the far-infrared part of the spectrum as well as the interband transitions.

\section{EXPERIMENTAL}

Self-standing SWNT films were prepared by filtration of a dilute solution [3] of laserproduced material [4]. The sample used for optical measurements was transferred to a graphite template with an approximately $4 \mathrm{~mm}$ hole. This sample geometry and the high transparency of the film allowed us to measure transmission with high reproducibility. We used two different spectrometers with liquid helium cryostats: in the FIR through MIR range a Bruker 113v spectrometer, in the NIR, visible and UV a modified PerkinElmer grating spectrometer. Spectra were obtained before and after heat treatment in vacuum at $1000^{\circ} \mathrm{C}$ (to remove $\mathrm{HNO}_{3}$ that $\mathrm{p}$-dopes the material; the procedure is hereafter referred to as "baking"). We examined three different samples. Samples 1 and 2 were of the same batch, one baked and one unbaked; sample 3 was from a subsequent batch, 
here data were taken on the same specimen before and after baking.

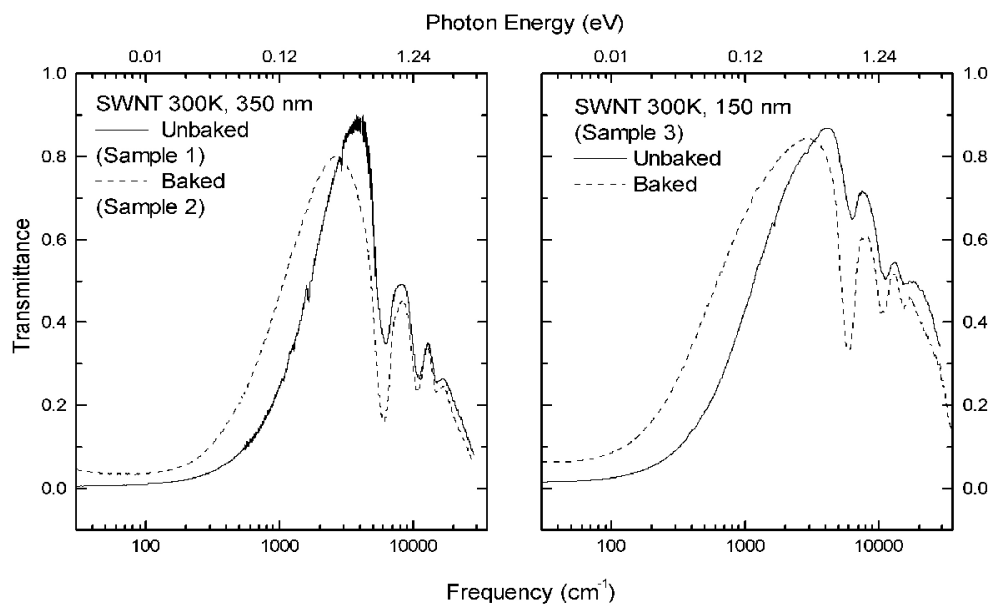

FIGURE 1. Transmission of SWNT films before and after baking.

\section{RESULTS AND DISCUSSION}

Figure 1 shows the transmission of the samples at room temperature. The films reach transmission values of up to 80 per cent around $3000 \mathrm{~cm}^{-1}$ and are highly transparent (above 30 per cent) throughout the whole midinfrared range. Baking increases transparency in the far infrared and decreases it somewhat at higher frequencies. For application purposes, obviously the featureless midinfrared region is of importance, but the information about fundamental materials properties is carried by the highly absorbing structures in the far and near infrared.

The transmission must be consistent with Kramers-Kronig relations, just as is the reflection, and the phase shift on transmission may be estimated from a Kramers-Kronig integral on $\ln T$, just as with the reflectance. After computing the phase, one may extract the complex refractive index (and all other optical constants) using equation (1).

$$
\sqrt{T} e^{i \Theta}=\frac{4 N}{(N+1)^{2} e^{i \delta}-(N-1)^{2} e^{i \delta}},
$$

where $\delta=\frac{\omega N d}{c}$. The sample thickness $d$ can be a fitting parameter as well, but in our case we used $350 \mathrm{~nm}$ for Sample 1 and 2 and $150 \mathrm{~nm}$ for Sample 3, obtained from AFM measurements.

We fitted the transmission data by a Drude-Lorentz model. In the fits to the optical conductivity $\sigma_{1}$ of Sample 2 (totally removed $\mathrm{HNO}_{3}$ ) one can clearly see a low frequency 
oscillator (Figure 2.). This phenomenon has been predicted, attributed to a secondary gap caused by the curvature of certain semimetallic tubes [5] or the symmetry breaking by the neighboring tubes in metallic nanotube bundles [6]. Ouyang et al. [7] have measured both gaps on individual nanotubes by tunneling spectroscopy; based on their results, we attribute the $8 \mathrm{meV}$ peak to a curvature-induced gap in low-gap semiconducting tubes. Consequently, we assign the broader Drude contribution to metallic (armchair) tubes. The sample dependence of the low-frequency behavior is probably caused by the fact that the small gaps are easily filled by extrinsic carriers even at minimal doping.



FIGURE 2. Fits to room-temperature optical conductivity in Sample 2 and 3.

Figure 3. summarizes the oscillator strength values for all measured temperatures in Sample 3. It is apparent that observable changes on dedoping occur almost exclusively in the narrow Drude part. This change is accompanied by a sharpening and strengthening of the high-frequency van Hove transitions, already shown by Hennrich et al. [8], as the valence bands of high-gap semiconducting tubes are filled by electrons. The oscillator strength shows little temperature dependence. Such behavior is expected in normal metals, however, we see also very weak dependence of the scattering rates on temperature. This is consistent with previous optical [1] and dc measurements and points to the possibility of exotic transport mechanisms in nanotubes.

\section{CONCLUSIONS}

We measured the transmission spectra of ultrathin SWNT films in a wide temperature and frequency range. The low-frequency part of the spectrum can be fitted with two components, which we attribute to armchair and low-gap semiconducting tubes, respectively. Optical signatures of hole doping can be observed only in the semiconducting 


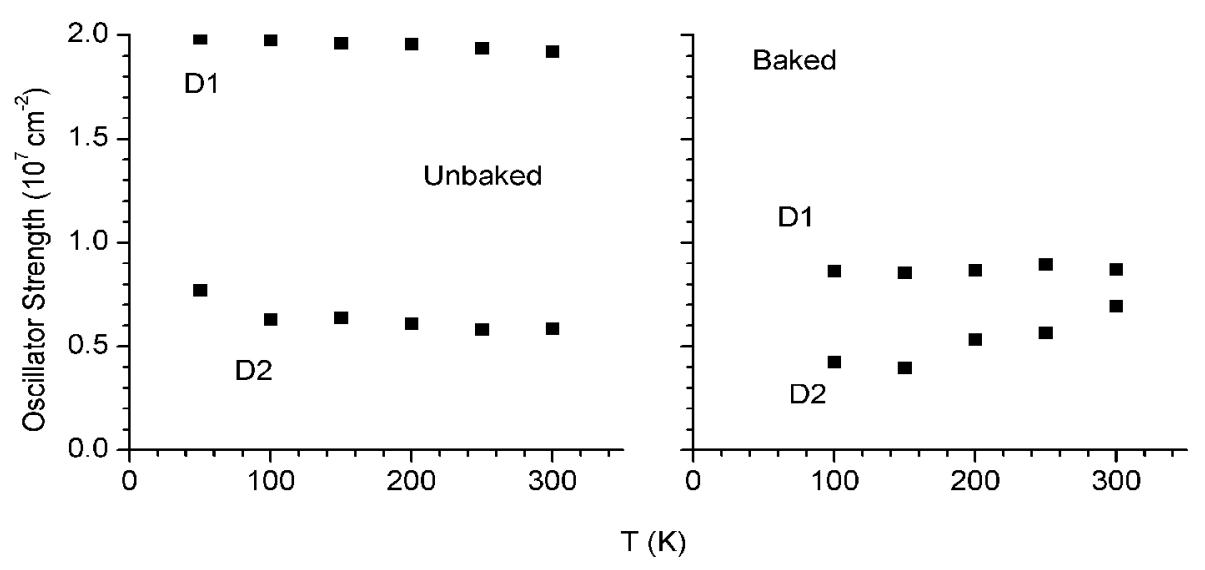

FIGURE 3. Temperature dependence of low-frequency oscillator strength in Sample 3 before and after baking.

tubes (both low- and high-gap). The temperature dependence of the optical functions is much weaker than what would be expected for conventional metals.

\section{ACKNOWLEDGMENTS}

This work was supported by the following grants: OTKA 034198, ARO grant DAAD1999-1-0316, NSF (ECS-0210574), US NSF-INT 9902050.

\section{REFERENCES}

1. Ugawa, A., Rinzler, A. G., and Tanner, D. B., Phys. Rev. B, 60, R11305 (1999).

2. Itkis, M. E., Niyogi, S., Meng, M. E., Hamon, M. A., Hu, H., and Haddon, R. C., Nano Lett., 2, 155 (2002).

3. Hennrich, F., Lebedkin, S., Malik, S., Tracy, J., Barczewski, M., H. Rösner, and Kappes, M., Phys. Chem. Chem. Phys., 4, 2273 (2002).

4. Rinzler, A. G., Liu, J., Dai, H., Nikolaev, P., Huffmann, C. B., F. J. Rodriguez-Macías, Boul, P. J., Lu, A. H., Heymann, D., Colbert, D. T., Lee, R. S., Fischer, J. E., Rao, A. M., Eklund, P. C., and Smalley, R. E., Appl. Phys. A, 67, 29 (1998).

5. Kane, C. L., and Mele, E. J., Phys. Rev. Lett., 78, 1932 (1997).

6. Delaney, P., Choi, H. J., Ihm, J., Louie, S. G., and Cohen, M. L., Nature, 391, 466 (1998).

7. Ouyang, M., Huang, J.-L., Cheung, C. L., and Lieber, C. M., Science, 292, 702 (2001).

8. Hennrich, F., Wellmann, R., Malik, S., Lebedkin, S., and Kappes, M. M., Phys. Chem. Chem. Phys., 5, 178 (2003). 